\title{
The distribution of landed property
}

\author{
Pavel Exner ${ }^{1,2}$, Petr Šeba ${ }^{2,3,4}$ and Daniel Vašata ${ }^{2,5}$ \\ ${ }^{1}$ Nuclear Physics Institute, Academy of Sciences of the Czech Republic, CZ-25068 \\ Rež near Prague, Czech Republic \\ ${ }^{2}$ Doppler Institute for Mathematical Physics and Applied Mathematics, Břehová 7, \\ CZ-11519 Prague, Czech Republic \\ ${ }^{3}$ University of Hradec Králové, Víta Nejedlého 573 CZ-50002 Hradec Králové, Czech \\ Republic \\ ${ }^{4}$ Institute of Physics, Czech Academy of Sciences, Cukrovarnická 10, CZ-18000 \\ Prague 8, Czech Republic \\ ${ }^{5}$ Department of Physics, Faculty of Nuclear Sciences and Physical Engineering, \\ Czech Technical University, Břehová 7, CZ-11519 Prague, Czech Republic \\ E-mail: exner@ujf.cas.cz, seba@fzu.cz, daniel.vasata@gmail.com
}

\begin{abstract}
The distribution of property is established through various mechanisms. In this paper we study the acreage distribution of land plots owned by natural persons in the Zlín Region of the Czech Republic. We show that the data are explained in terms of a simple model in which the inheritance and market behavior are combined.

PACS numbers: 05.65.+b, 89.65.Gh
\end{abstract}




\section{Introduction}

Quantitative study of human behavior requires reliable data and tools to analyze them. The latter improved dramatically in recent years when entries in various databases became available in a digital form. From the sociological point this opens way to new highly interesting empirical findings and theoretical endeavors to understand them.

One of the first publicly available records were the telephone books which gave an opportunity to study the distribution of surnames. Interpreting the results in a model setting, the recent records found in the telephone book are understood as a result of a long process of intermarriages and surnames inheritability, hence the surname statistics refers to social and population processes in the past [1]. In particular, it is known that the numbers of individuals sharing the same surname follows a scaling rule known as Zipf law. Let us arrange the surnames in the descending order with respect to the number of entries found in the telephone book, and let $N(1)$ denote the number of records found for the most frequent surname, $N(2)$ the number os records for the second widespread surname, etc.; then the Zipf law says that $\log (N(k)) \approx-c \log (k)$ with some constant $c$.

The mechanism of inheritance from a single parent appears not only in the social context: similarly to surnames it applies to nonrecombining alleles in the genom, hence it is not surprising that there is a close link between the surname distribution and the human genome [2]. Furthermore, such a situation is not restricted to surnames but applies also to groups of people sharing a common native language, to the species diversity in ecological systems and so on - cf. [3]. It demonstrates that cultural traditions are transmitted from the ancestors to the descendants through a process analogous to the genetic heredity and display a close parallelism between the cultural and biological evolution - recall that in the evolutionary psychology these mechanisms are studied under the name "memetics" [4, 5].

In the field of economics the power law distribution is traditionally named after

the classical work [6] of Vilfredo Pareto; it is used to describe phenomena such as the statistics of personal income, or the allocation of wealth in a steady society [7, 8], however, also fluctuations of the stock prices or the land estate display such a behavior [9, 10].

Our aim in this paper is to combine these two aspects into a single model capable of describing situations in which both the ancestor-descendant dynamics and the market behavior occur. Specifically, we will analyze the distribution of the sizes of land plots owned by individuals. It is clear that - in contrast to a corporative ownership - a natural person can acquire the land either on the real estate market or to inherit it. On one hand the land is transmitted from an ancestor his/her descendant similarly like surnames, on the other hand it is a subject to changes by acquiring/selling the land on the real estate market. We are going to demonstrate that such a complex human behavior can be reasonably well described by a simple mathematical model.

The empirical basis for our investigation are data obtained from the Czech Office for Surveying, Mapping and Cadastre describing the present status of the legal relations to 
real estate property in the Czech republic. This includes, in particular, the information about the sizes, types, geographical location, and owners of the individual land plots; here we focus on the statistical distribution of the plot sizes owned by individuals.

The idea to use cartographic data is not new. For instance, the information on the spatial structure of the urban networks has been used in [11 to analyze the distribution of vehicular flows. There are also direct attempts to model future developments of the land use - in particular in the urban regions - such an information is of value especially for the real estate developers. These models, however, are not simple - see, e.g., [12] for a cellular automaton approach. Our intention in the present work is more simple: we are going to describe the actual division of the surface of (a part of) the Czech Republic territory among individuals as a steady state resulting from a long-term process of real estate inheriting and trading. Needless to say, we do not care about the individual plot sizes, only about their statistical properties.

Let us describe briefly the contents of the paper. In the next section we present and discuss a simple land trading/inheritance model. The resulting statistical properties of the plot sizes will be then compared with the true cadastral data for the Zlín region in Section 3.

\section{A simple real estate trading/inheritance model.}

To motivate the model, note first that the Czech cadastral records, which we work with, do not contain the land plot history, and consequently, the past real estate transfers cannot be directly extracted from them. There are indirect clues that contain information on carried transfers: any conveyance of real estate for consideration is subject to the real-estate-transfer tax. On the other hand, the inherited or donated realties are liable to the estate and capital transfer tax. Unfortunately, this tax is levied on all assets which makes the information about inherited realties obscured. Nonetheless, the taxing information represents the process of realty conveyance in the metric of the current prices. Recall that the price metric was used, for instance, to analyze the mechanism leading to the crash of the Japanese land market at the end of eighties - see [13]. It does not say much, however, about the actual acreage of the plots and about its historical development. Another factor which makes its use questionable is that the land price is subject to unpredictable fluctuations; to quote an example from the same study, the mean price of one square meter of land in Japan increased in the years 1983 -1993 nearly sevenfold [13]. The dynamics of land prices exhibits a volatility which makes it difficult to describe - we refer to [14] and references therein for more details.

With these facts in mind, we will focus on the statistical properties of the acreage of the land plots (cadastral units). They represent better objects to study because the plot sizes change little in the course of time and do not yield to market fluctuations. Most often the whole plot is conveyed and its size does not change at all; changes of the plot size are rare and are always related to a new surveying. The latter is typically 
a complicated and costly procedure, which is one more reason why the acreage is not vulnerable to speculations.

We understand the recent records contained in the land registry as a description of a steady state resulting from a long series of land inheritance and land trading. The total acreage is preserved, of course, and can be only redistributed among the new owners. We are going to describe the steady state by an agent-based approach developed originally to model the wealth of closed economies - see [15] for a review. For the sake of simplicity we will use the most elementary version designed initially to describe the social stratification - see [8] - it is also known as the "inequality process".

As usual in such situation we use discrete time proceeding in steps typical for an ownership change; one can think of them as of generations. Let $S_{k}(n)$ denote the acreage of the cadastral unit $k$ at time $n$ and $S_{k+1}(n)$ be the the acreage of its geometrical neighbor, by that we mean that $S_{k}(n)$ and $S_{k+1}(n)$ have a common border. Then the model cadastral dynamics we propose proceeds to the next generation in the following way,

$$
\begin{aligned}
& S_{k}(n+1)=\lambda S_{k}(n)+a\left((1-\lambda) S_{k}(n)+(1-\mu) S_{k+1}(n)\right) \\
& S_{k+1}(n+1)=\mu S_{k+1}(n)+(1-a)\left((1-\lambda) S_{k}(n)+(1-\mu) S_{k+1}(n)\right)
\end{aligned}
$$

where $\lambda, \mu \in[0,1]$ are independent random variables with identical distributions and $a$ is a Bernoulli variable taking values $a=0,1$, each with probability $1 / 2$. The meaning of the dynamical equations (1) is straightforward. With probability $1 / 2$ we have $a=1$ in which case the size of the plot $k$ increases, $S_{k}(n+1)=S_{k}(n)+(1-\mu) S_{k+1}(n)$, while the size $S_{k+1}$ of the unit $k+1$ is reduced, $S_{k+1}(n+1)=\mu S_{k+1}(n)$. In other words, the cadastral unit $k$ of original acreage $S_{k}$ has incorporated a part of the neighboring land plot $k+1$. As a result, we get (after a surveying and introducing into the land register) two new cadastral units of acreages $S_{k}(n+1)$ and $S_{k+1}(n+1)$. With the same probability we have $a=0$ in which case the plot $k$ shrinks, $S_{k}(n+1)=\lambda S_{k}(n)$, with a part of it being incorporated into $k+1$ giving $S_{k+1}(n+1)=S_{k+1}(n)+(1-\lambda) S_{k}(n)$.

We are interested in the probability distribution of the steady state to which $S_{k}(n)$ converges as $n \rightarrow \infty$. A general mathematical result ensures that the limit of such a process exist and is unique - see [16]. It depends, of course, on the statistical properties of the variables $\lambda$ and $\mu$ and can be numerically evaluated by iterating the mapping (1) - cf. [17]. Moreover, in analogy with [18] one can argue that the convergence is fast coming close to the equilibrium in just a few generations, hence one can expect the model will be applicable to processes of land plot redistribution provided they run undisturbed for at least a century.

The variable $\lambda$ describes the land conveyance and contains in such a way the information about the generation dynamics (we refrain from mentioning the variable $\mu$ all the time since it has identical statistical properties). The mechanism is simple: assume that the owner of a cadastral unit has three offsprings. When he or she dies, the children become after the appropriate inheritance procedure co-owners of the cadastral unit with the one-third share each; the fact is recorded into the land register. We 
disregard the possibility that the heritage involves a more complex settlement leading to other proportions assuming that such effects will statistically cancel. Assume now that one of the descendants decides to individualize his or her share, i.e. to discontinue the co-ownership and to register the part as an individual belonging, or he/she decides to sell it. After the appropriate surveying the original size of the plot changes by the factor $1 / 3$, i.e. we have $\lambda=1 / 3$ in this case. It has to mentioned here that matrimony does not establish a joint property in the above judicial sense. A married couple acquires a land estate into a tenancy in its entirety. This is a different legal category and it is registered differently; we will handle it as a full ownership of the plot. on the other hand, a marriage separation leads to a co-ownership.

An instantaneous snapshot of all these processes is recorded in the cadastral database. On particular, we can derive from it the probability $p_{n, m}$ that a fraction $n / m$ with $m>n$ of the cadastral unit is in co-ownership. To take then into account the genealogical part od the land conveyance we use this information to define the appropriate distribution of the statistical variable $\lambda$ : we suppose that it takes the value $\lambda=n / m$ with probability $p_{n, m}$ whenever $m \geq 2$. We can leave out the case $m=1$ when the land plot is inherited as a whole, $S_{k}(n+1)=S_{k}(n)$, noting that the identity of the owner is not important.

The inheritance does not tell us the whole story, of course, since a part of the real estate is traded on the market. This concerns, in contrast to the above, the plots with $n=m=1$ having a sole proprietorship with $\lambda=1$. Such plots - being fully owned by a single person - are subject to free trading. For simplicity we will assume that the trading mechanism is completely random in full analogy with the closed economy models [15]). In other words, we suppose that on the subset of plots with $n=m=1$ the quantity $\lambda$ is a uniformly distributed random variable.

To summarize this part, we have formulated the model of exchange of cadastral units governed by the dynamical map (11). For units that are in a co-ownership of different persons the variable $\lambda$ equals to the corresponding share and enters the equations with the probability matching the relative appearance of the given share among all the cadastral records. On the other hand, for cadastral units have a sole proprietorship the variable $\lambda$ is random and uniformly distributed. In such a way if a piece of real estate is co-owned only the appropriater share can be traded, while those with a single proprietorship can be on the other hand traded without limit in a fully random way.

The corresponding dynamics can be now investigated numerically. One finds that the process converges fast, as we argued above, and the resulting equilibrium distribution is not sensitive to the initial state of the cadastre. In the next section that will compare the steady result of this linear model with the data and show that it leads to an amazing agreement with the acreage distribution extracted from the Czech Land Registry. 


\section{The results}

To begin with we note that if the random part of the dynamics (1) is omitted the land possession becomes fractalized. As a mathematical result about iterative maps with Bernoulli variables it was demonstrated not so long ago - see, for instance, [19]. The essence of the effect, however, was a fact well known to our ancestors and it was behind various juridical acts attempting to prevent a land structure disintegration. One can mention the so-called birthright edict - a part of the legal reform of the Habsburg Emperor Joseph II dated 1787 - which did not allow to split the land below 40 "measures", which is roughly 7.6 ha or 19 acres. This law was valid until the year 1869 when the rural land market - representing the random part of the dynamics (1) - became vivid enough and the edict became obsolete. The imperial act allowing the free divisibility and exchangeability of land (in Moravia, from which our data are taken, it was valid already one year earlier, in 1868) caused an unprecedented increase of the land exchange. For example, according to [20] in average 10\% of estates changed the proprietary relations yearly during the period $1869-87$.

What we find in the present cadastral data is thus a result of a process that started in 1868, some five generations ago. At the beginning it was marked by an boosted land exchange and the dynamics became steady only about a generation later. While these fact are documented, one may speculate about other factors having impact of the dynamics, in particular, changes in the population structure. It is obvious that that the natality and the number of offsprings living up to the adulthood were considerably higher at the beginning of the process, and as a result, the distribution of the land co-ownership was different from the present data. Unfortunately, the digitalized Czech land registry does not contain such a history record - all we see is a snapshot of the recent situation. In the light of these comments indicating how simplified our model dynamics (10) is, it is surprising and worth of attention how well do its result agree with the observed acreage probability distribution.

One more comment is needed before the results can be presented. We are dealing with all the plots contained in digitalized cadastral map with the exception of the build up areas. The reason for this methodical choice is simple: build up areas are places where building stand. In the cadastral map, however, we see only the ground plane, i.e. the projection of this building which does not tell us what the latter is like: it can be a simple one floor house as well as a high-rise construction. An information on the buildings is not a part of topographical data collection being contained in a separate registry, and some parts of the building (flats) can be traded leaving the ground plan unchanged. This is why we exclude the build up areas from the considerations and will discuss them in a separate paper.

For the comparison we have used the cadastral records of the Zlín Region, a territorial unit of the Czech republic of the area $3964 \mathrm{~km}^{2}$ and population of 590 thousand. We selected all the plots owned by individuals, excluding those owned by companies. It is clear that the plots owned in a co-ownership, or in the so-called 


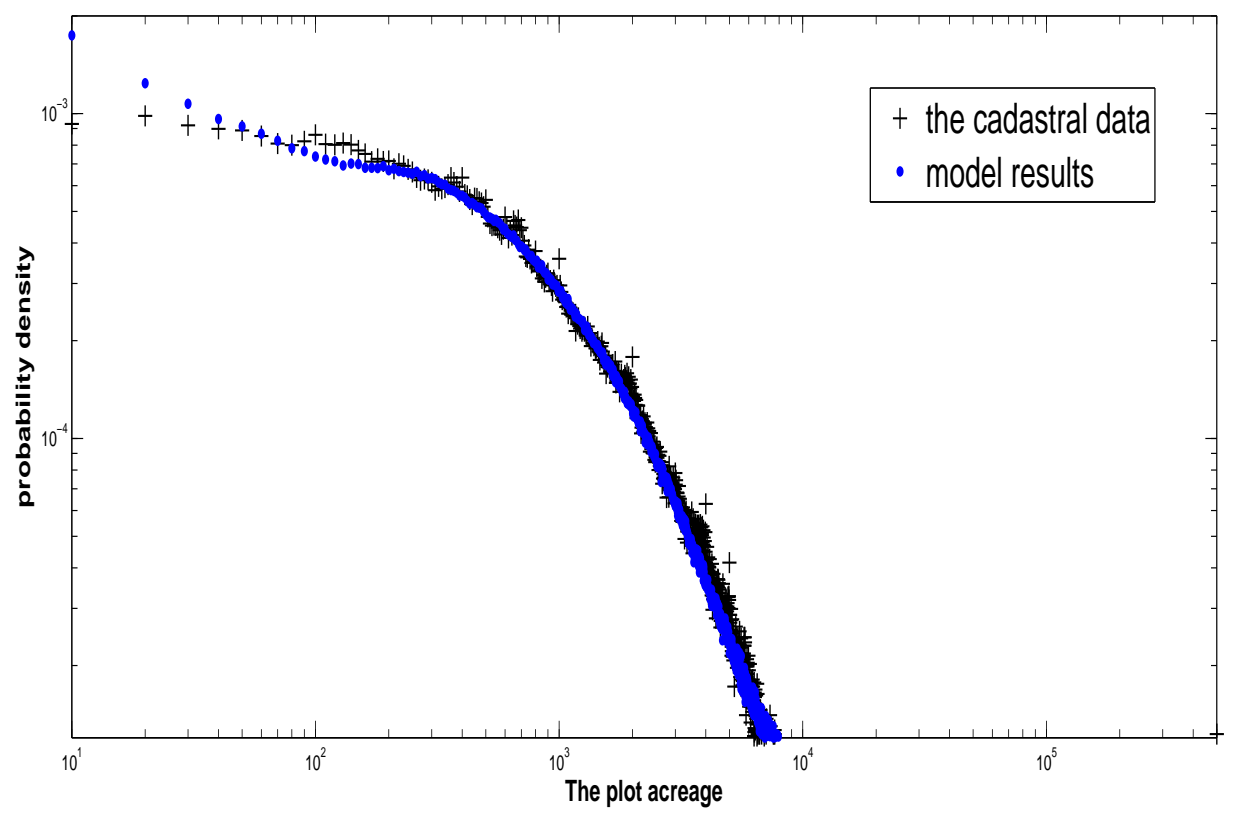

Figure 1. The probability density of the a given plot acreage found in the cadastral records (black crosses) is compared with the distribution obtained by iterating the map (11) (blue points).

undivided co-ownership of spouses are recorded repeatedly times in the data, hence the duplications were removed to get a meaningful size distribution. As indicate above all the build up areas were deleted from the sample. This left us with the collection of 1200121 plots which we could use to work out the size statistics and to evaluate the acreage probability density.

The same cadastral data were used to evaluate the probabilities $p_{n, m}$ of the particular shares $\lambda=n / m$ describing the plot co-ownership as described in the previous section. The map (11) was then iterated starting from the uniform distribution, $S_{k}(1)=1$ for all $k$. The result was subsequently rescaled to the mean which equals the mean plot size found in the cadastre and the two probability densities were compared; the result is plotted on the Figure 1.

We see that the two match perfectly; it is worth to stress that the model contains no free parameters. On the other hand, it is obvious that the data do not display the Pareto behavior. This fact is easily explained; it is enough to realize that the data represent the acreage probability of the plots and not the the probability of the individual belongings. The point is that one person can own more plots and their acreage (counting of course with the related co-ownership fraction) has to be summed. If we do this the Pareto behavior is restored as can be seen from the appropriate distribution plot on the Figure 2, 


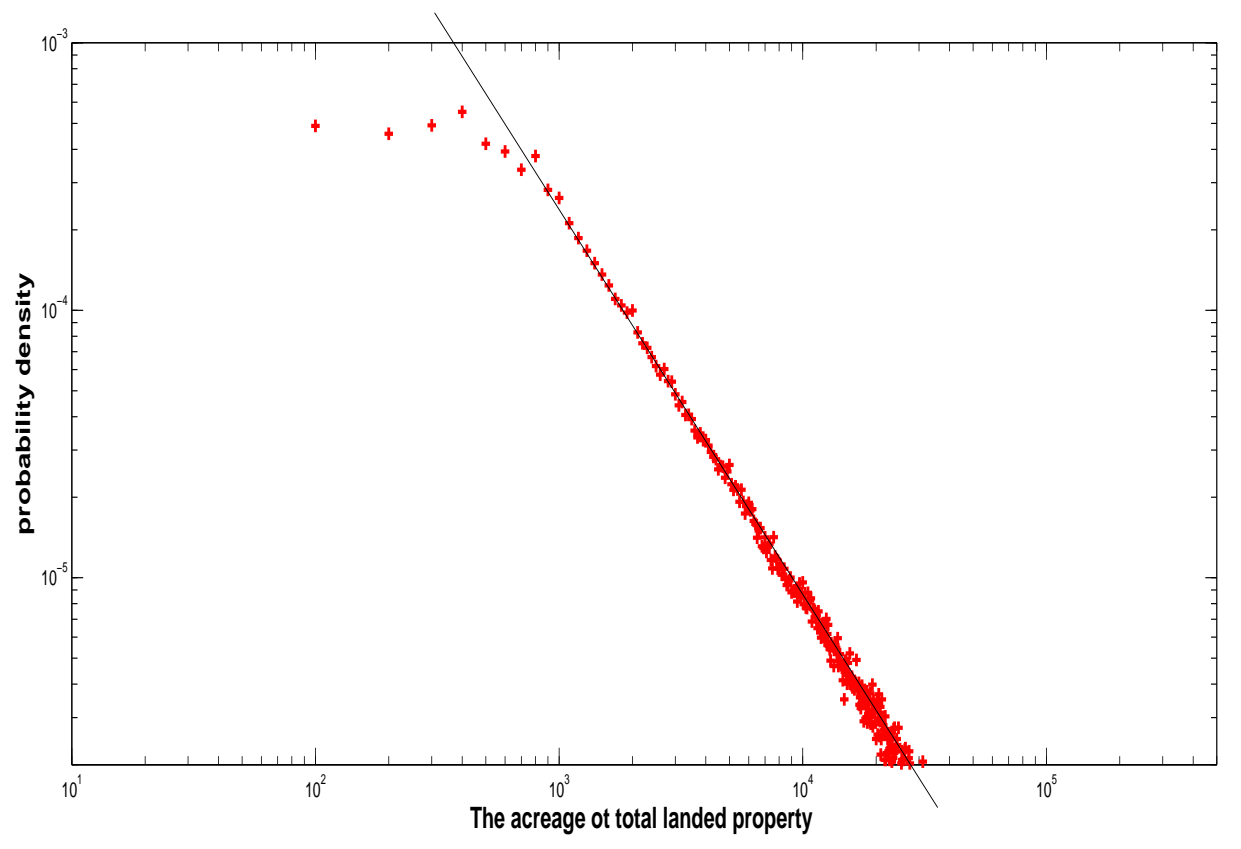

Figure 2. The probability density describing the acreage of the total landed property owned by individuals in the Zlín Region. The Pareto behavior is clearly visible

\section{Acknowledgments}

The research was supported by the Czech Ministry of Education, Youth and Sports within the project LC06002 and the Grant Agency of the Czech Republic No. 202/08/H072. We are indebted to Helena Šandová and Petr Souček from the Czech Office for Surveying, Mapping and Cadastre for the help with acquiring the data.

[1] Derrida B, Manrubia S C and Zanette D H 1999 Phys. Rev. Lett. 82 1987-1990

[2] Manrubia S C and Zanette D H 2002 J. Theor. Biology 216 461-477

[3] Sole R V and Alonso D 1998 Adv. Complex Systems 1 203-220

[4] Dawkins R 1976 The Selfish Gene (Oxford: Oxford University Press) chap. 12

[5] Blackmore S 1999 The Meme Machine (Oxford: Oxford University Press)

[6] Pareto V 1897 Course d'economie politique (Lausanne: Rouge)

[7] Huang Z F and Solomon S 2002 Physica A 306 412-422

[8] Patriarca M, Chakraborti A, Heinsalu E and Germano G 2007 Eur. Phys. J. B 57 219-224

[9] Sornette D 2002 Physica A 309 403-418

[10] Kaizoji T 2005 Physica A 347 575-582

[11] Laemmer S, Gehlsen B and Helbing D 2006 Physica A 363 89-95

[12] White R and Engelen G 2000 Computers, Environment and Urban Systems 24 383-400

[13] Kaizoji T and Kaizoji M Physica A 344 138-141

[14] Leung C K Y and Chen N K 2006 J. Real Estate Research 28 293-320

[15] Samanidou E, Zschischang E, Stauffer D and Lux T 2007 Rep. Prog. Phys. 70 409-450

[16] Devroye L and Neininger R 2002 Adv. Appl. Probability 34 441-468

[17] Knape M and Neininger R 2008 Methodol. Comput. Appl. Probability 10 507-529 
[18] Exner P. and Šeba P 2008 J. Phys. A: Math. Gen. 41045004

[19] Baron M and Rukhin A L 2001 Stat. and Probab. Lett. 55 29-38

[20] Urban O 1978 Capitalism and Czech Society (in Czech; Prague: Svoboda) 